\title{
Teaching death: exploring the end of life in a novel undergraduate course
}

\author{
David Kulp ${ }^{1}$, Lynn O'Neill², Tammie Quest ${ }^{2}$, Susan Tamasi ${ }^{3}$, Kim Loudermilk and Arri Eisen ${ }^{4^{*}}$ (i)
}

\begin{abstract}
Western culture discourages discussion of death and dying, especially with healthy emerging adults. Yet, research shows that engaging this population in conversations about death and dying is empowering and important for young people's decision-making around and understanding of the end of life. We show that students are indeed ill-informed on such issues but that they desire to learn more. We describe and assess a pilot undergraduate course in palliative care addressing this need, and we demonstrate its success in engaging and educating students using pedagogical approaches built to develop a social and intellectual community of trust.
\end{abstract}

Keywords: Advance care planning, Palliative care, Ethics, End-of-life, Higher education, Interdisciplinarity, Pedagogy

\section{Introduction}

Western culture perpetuates the myth that conversations about palliative care and death should only occur at the end of life. Palliative care is the subspecialty of medicine that provides holistic care for patients living with serious illness to ultimately improve quality of life for patients and their families. Typically, such communication only happens when it is too late-after life-limiting conditions present. However, dialogue about these difficult topics proves to be a crucial component of development in emerging adulthood and is especially important in this time of pandemic.

Palliative care specialists help to navigate these questions through sustained goals of care conversations with the patient and family starting at the point of diagnosis. Rather than engaging palliative care at the brink of death, palliative care has become integrated into the curative care plan for seriously ill patients, aiming to improve quality of life during treatment. As the conditions worsen and patients become increasingly ill, palliative care shifts to symptom-focused pain management and end-of-life

\footnotetext{
*Correspondence: aeisen@emory.edu

${ }^{4}$ Department of Biology and Institute for the Liberal Arts, Emory University, Atlanta, GA 30322, USA
}

Full list of author information is available at the end of the article care, as well as psychosocial and bereavement support for the patient and family members. Emerging adultsdefined as 18- to 23-years-old for the demographic of this study (What Should the Age Range Be for AYA Oncology?, 2011) - are not immune to serious illness, and many eventually become substitute decision-makers for their loved ones, forcing them to confront end-of-life questions for themselves or someone they care about. Early engagement-even prior to diagnosis of a serious illness-therefore may prove effective in destigmatizing palliative care and end-of-life care specifically.

Each year, more than one million adolescents and young adults themselves die from preventable causes, according to a recent 2017 study by the World Health Organization (WHO) (Chriscaden, 2017). Car-related accidents remain the leading cause of death for emerging adults globally, and suicide competes as a close second, its prevalence increasing steadily since 2007 (Howard, 2018; World Health Organization, 2018). And with a pandemic sweeping the world over the last year-including recent studies alluding to potential implications for the lives of young people-the fear of chronic and potentially life-threatening illness is palpable (Arnold et al., 2020; Cha \& Janes, 2020).

To decrease anxiety and fear about the end of life, the American Academy of Pediatrics and WHO recommend

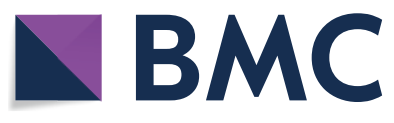

(c) The Author(s) 2021. Open Access This article is licensed under a Creative Commons Attribution 4.0 International License, which permits use, sharing, adaptation, distribution and reproduction in any medium or format, as long as you give appropriate credit to the original author(s) and the source, provide a link to the Creative Commons licence, and indicate if changes were made. The images or other third party material in this article are included in the article's Creative Commons licence, unless indicated otherwise in a credit line to the material. If material is not included in the article's Creative Commons licence and your intended use is not permitted by statutory regulation or exceeds the permitted use, you will need to obtain permission directly from the copyright holder. To view a copy of this licence, visit http://creativecommons.org/licenses/by/4.0/. 
having conversations with terminally ill children and emerging adults about decision-making as soon "as they are developmentally and emotionally ready" (Pao \& Mahoney, 2018; Wiener et al., 2012, 2013). Despite these data, end-of-life conversation is largely avoided with healthy emerging adults.

Colleges and universities are uniquely situated to facilitate such conversations through carefully designed coursework and pedagogical models effective for approaching divisive topics. Here we describe and assess the efficacy of one model to approach this conversation: a palliative care curriculum piloted for undergraduates that is part of a broader project to develop strategies for such teaching that could be used by a variety of academic institutions. Early interventions in educational settings-relying on resources typically used in advance care planning (ACP) - may prove important in empowering healthy emerging adults to make well-informed decisions about end-of-life care (Kavalieratos et al., 2014, 2015; Tripken \& Elrod, 2018). There is currently a wealth of literature related to young adults with serious illness, including the importance of end-of-life conversations with young adults suffering from terminal illnesses (Lyckholm \& Kreutzer, 2010; Wiener et al., 2012), shifting the emphasis to include families and terminally ill emerging adults in end-of-life planning (Jalmsell et al., 2015; Kon, 2006; Robinson et al., 2019), enhanced provider communications training (Billings, 1997; Head et al., 2016; Odeniyi et al., 2017; Tulsky, 1998), psychological conceptualizations of death in emerging adulthood (Dehlin \& Reg, 2009; Duke et al., 2011; Noppe \& Noppe, 2004; Slaughter \& Griffiths, 2007; Willis, 2002), and the newly established standards of care in Western medicine (Pao \& Mahoney, 2018). Research on such communication and engagement with healthy emerging adults, however, is lacking. The implementation of a novel pilot undergraduate palliative care-oriented course aims to bridge the gap in communication with healthy emerging adults about death and dying. Ultimately, we sought to increase both the pragmatic awareness of palliative care planning tools and to understand the influence of discussion on student self-identification with sensitive end-of-life conversation through an undergraduate curriculum focused on humanistic investigations about death.

\section{Our university in context: the impetus}

\section{for an undergraduate palliative care curriculum}

Emory University is a private, mid-sized, Research I university located in the southeastern United States. It houses nine colleges and professional schools, including schools of law, medicine, public health, and business, as well as an undergraduate college, creating an especially rich environment for interdisciplinary education. The undergraduate college hosts a unique interdisciplinary studies program, dedicated to furthering interdisciplinary pedagogical teaching and providing a natural home for the development and implementation of a curriculum engaging death, dying, and palliative care that integrates so many diverse fields.

The curriculum was developed as part of a broader initiative to nurture a community of interdisciplinary students and faculty, and to discuss and expand on issues of death and dying at our university. Many faculty members address aspects of death and dying in their courses and research, but rarely engage with one another around these issues. A larger effort was led by the instructors for this course (a geneticist, an expert in American cultural studies, and a student using the course as a focus for his honors thesis) together with palliative care physicians in our institution's medical school to integrate discussion about death and dying. These leaders convened regular meetings of 25 faculty from 17 different departments. Complementary to the pilot course itself and the conversations with faculty across the institution, we linked the course to public seminars sponsored by the interdisciplinary program and led by faculty from this broader community. Several of these faculty members visited the pilot course to discuss their areas of expertise and were resources for students' course research projects. Our course and its related activities are now being developed as a model for others through the newly developed, interdisciplinary Undergraduate Palliative Education Program. In fact, a consortium of ten other similar institutions has now been recruited to further cultivate and implement undergraduate education in death, dying, and palliative care across the USA (https://www.palliative undergrad.org/).

\section{Feasibility study: end-of-life tools and terminology}

To corroborate the theories of engaging healthy undergraduates in end-of-life education, we conducted a feasibility study among undergraduates at our university in Spring 2019 to better understand the familiarity of healthy college-age emerging adults with end-of-liferelated tools and their comfort level in discussing death and dying. The study gauged familiarity with ACP tools and terminology, including advance directives (AD), durable power of attorney for healthcare (DPOAH), and do-not-resuscitate orders (DNR). The survey was posted to two moderated Facebook groups. IRB approval was granted retroactively under the IRB protocol submitted in Fall 2019 for the pilot course research study (see "Appendix A").

Responses $(n=67)$, collected anonymously and representing students in all four years of undergraduate education, indicated that conversations and knowledge about 
end-of-life are very limited. Over $80 \%$ of respondents reported never speaking with their parents/legal guardians about ADs, and only $18 \%$ of students had previously learned about ADs in academic courses. A mere $8 \%$ of students had completed an AD. The numbers are just as striking for completing a DPOAH (11\%), and no respondents reported speaking to a primary care physician at any time about AD and DPOAH.

Respondents were also asked about their comfort level discussing death. Nearly two-thirds reported having no prior conversations with their parents about their parents' wishes for the end of life. Eighty-two percent of students reported never having spoken to their parents about their own wishes for the end of life. Notably, half of those surveyed thought end-of-life information and discussion should be incorporated into college curricula.

A limitation of the feasibility study is that the survey was collected with an assumption that the participants were healthy. Emerging adults who suffer from lifelimiting conditions often have significant contact with physicians over the course of their illness. Having such close interactions with specialists increases the chance of familiarity with end-of-life-related tools and terminology. Additionally, though participants were asked in the feasibility study if they learned about certain tools in academic settings, participants were not explicitly asked if they were pursuing careers in health professions. Students interested in medical or nursing school, for example, may have more exposure to medical terminology than business or liberal arts students, influencing their responses. Lastly, the pool of respondents was limited to undergraduates at one institution.

\section{Pilot course: a novel pedagogy}

Armed with the knowledge garnered from the feasibility study, we designed and taught Science and the Nature of Evidence: Death, Dying, and Finding Meaning in Fall 2019. The course was a semester-long, general education course within an interdisciplinary program that offers such courses to students from any major. The program also allows students to design their own interdisciplinary majors.

\section{Course recruitment}

Science and the Nature of Evidence is taught every semester within the interdisciplinary institute at our university. Students register through the centralized university system. Given the interdisciplinary goals and nature of the course, recruitment is intentionally cast widely throughout the university through personal and interdepartmental emails. Additionally, the course fulfills several general education requirements-a stipulation of graduation from our university-thus organically driving student recruitment. Some students with specific interests in palliative care and medicine were approached. At the start of the semester, after registration, enrolled students were informed about the nature and topic of the class under the new subtheme of death and dying. Students were provided with an opportunity to withdraw from the course during an add/drop/swap period. Throughout recruitment and enrollment, the pilot course was intentionally kept small (13 students), both because of the sensitive nature of the material and because it was being taught for the first time. The pilot course was ultimately taught in-person on our university's campus.

\section{Community of inquiry model}

Notwithstanding the small pilot size, the key theories, themes, and pedagogies that drove and emerged from the pilot are adaptable to larger courses. We used the Community of Inquiry model as a theoretical framework for the course (Garrison \& Akyol, 2013). The model describes a "collaborative-constructivist" educational experience best illustrated by a Venn diagram of three interlocking circles Garrison calls social, cognitive, and teaching presence. Social presence describes how students and teachers identify and create trust and effective relationships within the classroom community. Teaching presence refers to substantive pedagogies that result in meaningful learning. Cognitive presence is defined as students' construction of meaning via reflection and active engagement in learning.

Though the Community of Inquiry model provided the framework for our pedagogical method, we developed three driving themes, mapped alongside and at the interstices of the overlapping circles of the model, for our pilot course: (1) we focused on creating a community of trust, at the "setting the climate" intersection, where social and teaching presence meet; (2) we used personal belief and experience, complemented with experiences from experts from many disciplines through memoir and guest lecturers in the "selecting content" intersection between cognitive and teaching presence; and (3) course instructors modeled the personal and pedagogical methods for the students to facilitate the students' own original research and peer teaching-at the "supporting discourse" intersection (Fig. 1).

\section{(1) Setting Climate: A Community of Trust}

Crucial to the course, especially given our engagement with the topics of death and dying, was the focus on creating and sustaining a trusting social and intellectual Community of Inquiry. We used two pedagogical strategies toward this end: personal statements and checkins. Rather than being "add-ons" or touchy-feely extras, 


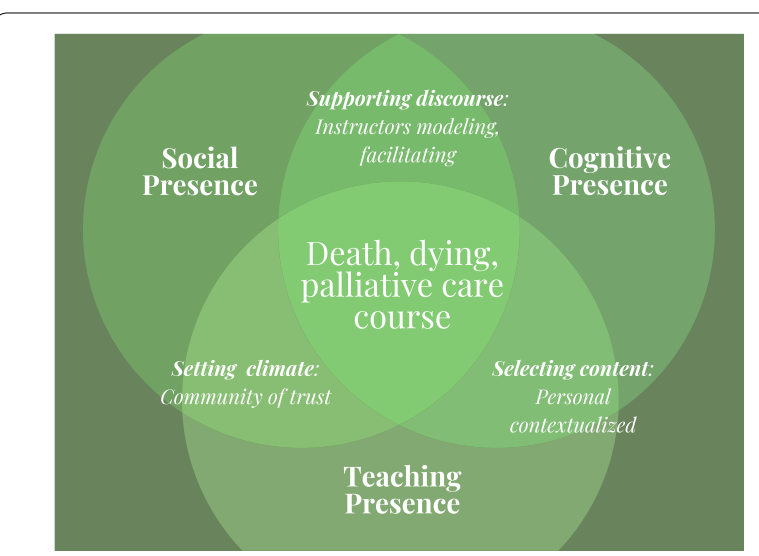

Fig. 1 The Death, Dying, and Finding Meaning course was driven by and fits into the Community of Inquiry conceptual and pedagogical model (Garrison \& Akyol, 2013)

these community-building pedagogies were integral to the course, as much a part of the content and evidence welcomed and wrestled within the class as the more traditional academic content we included in the course. These pedagogical approaches inevitably helped shape new content by pointing the students to topics to pursue further when they took on more intellectual leadership in the second half of the course.

\section{Personal statements}

Each of the first few class sessions began with 2-3 class members (beginning with the teachers on the first day of class) introducing themselves through personal statements-open-ended, one-page, read-aloud introductions that are not turned in and give the authors an opportunity to say who they are, why they are in this course at this moment, and to address any relevant experiences or ideas related to why they believe what they do about science, evidence, life and death. Such thick introductions quickly engage individual experience, enhance deep relationship building and help set the tone of the course. We made clear that details of personal statements were to be kept in the classroom. Beyond being effective introductions, many of the ideas and stories raised in these introductions naturally segued into further and more general discussion that day or were referred to throughout the semester, becoming touchstones of our classroom intellectual community.

\section{Check-ins}

Check-ins, while less formal than personal statements, were used throughout the semester to encourage students near the beginning of most class sessions to search for and voice connections they had made between class content and their daily lives. These oral reflections also gave students a moment to transition into the classroom frame of mind. For example, students would connect a class reading to their experiences with their grandparents in assisted living facilities or bring up information learned from other courses-past and present-that influenced their understanding of the material presented in the course.

Check-ins also provided a safe space for students to be vulnerable, for introspection and for emotional support. As the semester began, a second-year student who was not in the class lost their life in a presumed suicide. Some of the students in the course were close to this individual. The opportunity to share their personal experienceshow death intimately affects even college students, still presumed to maintain their "innocence"-was an eyeopening and centering experience for students and faculty in the course. After a student's sibling tragically died during the semester, their personal experience inevitably impacted the trajectory of the course content once again. Check-ins offered the opportunity to draft messages of support for the student and their family. Though such tragic events occur naturally in the lifecycle of an adult, discussing them in class with undergraduates in the context of scholarly course material was an important component of the course. Ultimately, check-ins offered the faculty an opportunity to shape content around certain stressors and better gauge the "state" of the classroom.

(2) Selecting Content: The Personal Contextualized in Interdisciplinary Scholarly Expertise

The second goal and theme of the course was to use the environment of trust established to contextualize the personal within the fabric of scholarship and expert experience in issues involving death, dying and palliative care. Here we employed three pedagogies: (1) engaging class guests through the rich interdisciplinarity both of the students themselves and of the community of expertsincluding palliative care physicians and others-at our institution; (2) reading and discussing relevant texts by other experts; and (3) assigning final individual research projects, presented both in writing and as a visual poster to the entire class.

\section{Interdisciplinarity}

Interdisciplinarity enriches and broadens socio-intellectual engagement. Our course was developed and taught by a biologist and a humanist, and it enrolled a variety of majors, from business to music, linguistics to American Studies, and anthropology to biology. Palliative care physician collaborators attended sessions of the course to teach both formally and informally about the foundations 
of palliative care and hospice medicine. A Tibetan Buddhist monk discussed practices from his tradition around end-of-life and reincarnation. He focused specifically on Tukdam, a meditative practice during the period of death of a Buddhist master that ultimately results in reincarnation according to traditional Buddhist philosophy. Another physician visited and highlighted philosophical and practical differences between intensive and palliative care, and provocative ideologies about a physician's duty to a dying patient. A director of our on-campus art museum led an exploration for the students of the permanent collection of artworks from ancient Egypt, Nubia, and the Near East; Greece and Rome; the Americas; and Africa, much of it relating to funeral and burial practices, and other cultural rituals at the end of life. This tour culminated in a dinner during which students and faculty reflected on what they had learned in the museum and integrated that knowledge with previous discussions in the class.

\section{Expert texts and discussion}

Often, the personal, the "first person," is intentionally excluded from academia, especially within the sciencesdismissed as an inadequate form of evidence. To put this theme into pedagogical practice here, as discussed above, we encouraged the inclusion of the personal as a foundational element of our course. We demonstrated the value of such expression by having explicit pedagogies that called for it and also by featuring it in the other types of evidence and texts explored: two of our main texts had significant elements of the personal-When Breath Becomes Air by Paul Kalanithi and Being Mortal: Medicine and What Matters in the End by Atul Gawande. A third text, The Cure Within: A History of Mind-Body Medicine by Anne Harrington, places the personal into historical and medical context.

\section{Final projects in a poster session}

The course culminated in a two-part research project consisting of a paper and a communal poster presentation. Building on the inclusion of the personal, trust and other pedagogies utilized in our Community of Inquiry model, we encouraged the students, in their final research projects, to include themselves and use their own experiences and ideas as evidence mined and integrated with their primary and secondary sources and consultations with faculty experts. Beginning on day one of the course, students brainstormed with peers and instructors about potential project topics during class time. As the ideas evolved, the teaching team and course peers gave feedback and shared their insights. Through the projects, students brought together both content and methods from more than one discipline. In brainstorming with peers, both the poster and related paper were enriched as students exchanged ideas and perspectives from their own disciplines. Then, to exhibit their findings and to test their ideas as they were developing a final written version of their projects, we hosted a public poster session at which students presented among themselves, the teaching team, and visiting faculty.

Final projects were diverse, interdisciplinary, and distinctive, often exploring a new angle on a subject with which the students were already familiar from their personal experience or scholarship: the psychological impact of trauma and torture, featuring interviews with victims the student knew; social perception of death as driven by divisive language used to describe abortions, drawing on the student's own conversations with women considering abortion; life and death traditions among the Gullah (an African American community in the Lowcountry region and Sea Islands of the U.S.); and identification of KüblerRoss grief stages in near-death experiences, among other topics. One music major focused on the relationship of music theory and the end-of-life trajectories that palliative care physicians had discussed earlier in the course. Grounding their final paper in an interdisciplinary medical and historical context, they asked how "historians' and palliative care doctors' common methods may be applied to the analysis of musical composition."

\section{(3) Supporting Discourse: Modeling, then Facilitating}

The last driving component of our course-instructors modeling teaching and learning and then facilitating the students in the same practices-is tightly connected to the other two interstices in our Community of Inquiry model. How better to build community than for the leaders to also get in the trenches? After learning from the instruction of the teaching team, the students became the leaders themselves in a safe and supportive environment with rigorous feedback and guidance. The course instructors accomplished this through, as noted, introducing themselves via personal statements on the first day of class; leading class sessions the first half of the course and then facilitating the same by the students the latter half; discussing their own scholarship in class and then carefully monitoring students' research project ideas in-class and through email and one-on-one personal meetings. The result was creative and immersive student-to-student learning inside and outside of the classroom.

\section{Teacher- and student-led learning}

For the first half of the course, teachers led sessions that grew from assigned readings featuring the major course texts; in the second half, students, adapting pedagogical strategies modeled by the teachers, led discussions 
on relevant topics of their choice. The first half had four sections-(1) Is science right?; (2) Memoirs about dying; (3) Dying across different cultures; and (4) The biology of death. Several core questions were explored throughout the course: "Why do you believe what you believe? What are the roots of those beliefs? What kinds of evidence do you accept, consciously or not?" All three books we read in the course relate to science, evidence, death, palliative care, and ethics, and collectively drove class dialogue. Pedagogies that teachers used to model engagement with these texts included small-group discussions based on questions about the texts that related to course themes, followed by full-class discussion; excerpts from movies, interviews or other media linked to readings to catalyze discussion; in-class debates in which students were randomly assigned to argue one side of contentious issues; and story circles, an activity in which students share stories to "engender appreciation for the unique intellectual, emotional, and spiritual qualities of each participant" through intentional listening (Roadside Theater, 2014).

For the second half of the course students in preassigned groups of two or three each led one session of class learning, selecting their own topics, drawing for inspiration on the personal statements, check-ins, and readings from the first half. This approach both allowed students the experience of peer-teaching and the class to continue exploring issues related to death and dying that students wanted to explore further.

A week prior to their sessions, each group discussed their proposed topic and pedagogical approach with the instructors for insight and approval and provided a short pre-class assignment for their peers. These sessions ranged in themes from spirituality and religion in endof-life care to cultural differences in funeral and burial practices; from complexities of pediatric palliative care to human reactions related to pet death; from ethics of physician-assisted death to unethical notification and communication of prisoner deaths; from evolution of care of older adults in the U.S. to ethics of life-support and invasive life-saving procedures.

In the student session about the role that spirituality plays in medicine and how it influences practices after death, the discussion leaders encouraged peers to think about their own future funeral and those of their friends and family: "Have you attended a funeral? Do you believe this process was intended to support the deceased or comfort and soothe the bereaved in their acceptance of death? How would you ask your loved ones to plan your funeral? Where would it take place, who would attend, who would speak?" The readings, in this case, enhanced the conversation, bringing in evidence and current studies that show the impact of religion on nurses and physicians involved in end-of-life care. Many of these student-initiated topics evolved and were integrated into final projects.

\section{Evaluation}

After the feasibility study demonstrated the potential need and desire for a palliative care education and dialogue in an undergraduate environment, we developed and piloted just such a curriculum. While the feasibility study conducted in Spring 2019 assessed awareness of end-of-life planning tools, we sought to investigate qualitative themes that emerged from an intentionally designed curriculum. Grounded in the Community of Inquiry model-integrating a three-pronged social, cognitive, and teaching presence-we used surveys to elicit quantitative and qualitative feedback from the students to evaluate the effectiveness of the pilot course (see "Appendix B").

At minimum, the course raised awareness of key terms and definitions among students. None of the respondents $(n=7)$ reported having had previous conversations with parents or legal guardians about their personal wishes for the end of life. As hoped for, following completion of the course, the number of students reporting knowledge of all three end-of-life tools-ADs, DPOAHs, and DNR orders-increased dramatically. For example, twice as many reported knowledge of AD after taking the course than reported having such knowledge beforehand. Within our theoretical model, this enhanced awareness was predicated on the successful implementation of a community of trust within which to discuss sensitive topics relevant to end-of-life care.

Student response, however, moved far beyond awareness of terms and practices. One of the cornerstones of our Community of Inquiry theoretical model was facilitating a space for cognitive presence-a place in which students construct meaning through personal reflection. When asked what drew them to the course, students mentioned (1) that they saw it as an opportunity to discuss death and dying-topics rarely discussed in college classrooms, (2) curiosity about how diverse students make meaning of life and death, and (3) that death is a "universal" and shared experience, so actively contemplating and discussing it "can be liberating." Our model thus successfully integrated academic awareness of endof-life planning and palliative care with personal reflection and engagement.

None of the respondents reported a strong understanding of palliative care prior to taking the course. When prompted to reflect on their definition and understanding of palliative care and its core tenets after the course, a common theme emerged among responses. Steeped in our interdisciplinary pedagogical approach to teaching, students reported a grasp of 
palliative care as a holistic biopsychosocial-spiritual model of care for patients of all ages suffering from lifelimiting chronic and terminal illnesses, and that palliative care physicians are focused on "much more than diagnosis and treatment." All respondents recognized the importance of this holistic care in acknowledging patient wishes at the end of life. Engaging with material from various fields-including material sciences, humanities, medicine, and social sciences-proved critical to holistic student understanding through which our Community of Inquiry model's emphasis on teaching presence flourished.

The two themes of the engagement and use of the personal and discussing and sharing that personal in the context of a trusting academic and social community recurred often in survey responses. Students were prompted to reflect on their personal experiences through the course and their comfort with end-of-life issues, including their personal reflections on death and dying. The majority of respondents (5/7) reported being comfortable talking about death and dying following the course. Students commented that the engagement of the personal in the course enabled them to confront finitude more consciously: "I would say that I have always been aware (in the back of my mind) that I was mortal, but taking the class and thinking explicitly about my own death definitely brought it to the forefront." Rooted in the modeling, then facilitating model, for many students the course served as the opportunity to "experience death more closely" without direct exposure in a clinical environment. Ultimately, the "class did not fail to remind me that life is finite... and I definitely think this class has heightened my awareness of my own mortality and fragility," as one student noted. However, rather than expressing fear toward this newly found awareness of mortality, students collectively expressed that discussing death and palliative care directly and acknowledging the fragility of human existence "gives life meaning." The teaching team ultimately successfully facilitated open and constructive dialogue with students, highlighting the importance of trust as explained in our Community of Inquiry's focus on social presence.

Another student reflected that, while the course did not impact their own awareness of mortality, it encouraged them to expand the personal into the context of the community of their peers within the course and beyond, to be "more observant of the way others deal with death outwardly, whether this is through social media, the services they hold, anything they do after [a] death in relation to that person's life." Similarly, a student explained that the course enhanced their own feelings of mortality "through events in my life that coincided with the course." Another reflected that the class has "made me more aware of aging, specifically in relation to my parents and grandparents." The course clearly encouraged more dedicated awareness of mortality, contextualizing personal experience within our academic pedagogy adapted to the Community of Inquiry model.

Students were asked directly about the influence of the course outside of the classroom-the impact on their interactions with peers and family. Many noted a newly found openness to thinking about and engaging with the topic directly among friends and family, for some even leading to behavioral changes. One student reflected that the course "made me think about raising some of the topics with my partner" that they "would've [not] thought about otherwise" before taking the course. Another noticed that they had made a conscious effort to spend "more time with my grandparents" after taking the course-a reflection of their reading and class discussion of Gawande's Being Mortal. Others again expanded the effects felt and knowledge gained from the course into the context of their daily interactions with the greater community:

...my sensitivity and respect for the elderly in my life has grown immensely, seeing as they have often told me they have been forced to confront the themes we have discussed in our class and I have now, at the least, begun to grapple with how shocking and challenging that can be. Furthermore, this course has cultivated in me a desire to volunteer at hospice and more deeply understand those 'editorial' moments that people can experience at the end of their lives.

One student reflected that the course enabled them to think more deeply about their lived experience, to think "critically about the afterlife, my [religious] views, finding meaning, [and] the process of death," and that they "found the discussions in this class to be soothing... while the material of the class was certainly heavy, I enjoyed taking time out of my week to discuss a concept or text surrounding death. I never would do that without this class." While faculty modeled teaching for student-led discussions, students facilitated and engaged on a deeper level than expected-re-evaluating, shifting, and implementing new behaviors and attitudes into their unique lived experiences.

When asked about what was most surprising about the course, respondents reflected on the depth and diversity of personal experiences that students shared publiclythe importance and impact of a trusting community: "I was consistently surprised both by how personal we were asked to get in this course and [by] how much I enjoyed the ensuing effects of doing so in the class." The personal statements served as a moment for reflective observation. Vulnerable introspection on concrete life experiences 
leveled the playing field, allowing students to recognize their collective encounters with death and trauma across the lifespan: "I was surprised by how diverse the experiences in our class [were] in relation to death and trauma through [the] personal statements. People go through some tough stuff in their lives and you wouldn't be able to notice it unless you truly got to know them, or took a course like this."

Though the personal nature of the conversation may be off-putting for some, death is seemingly relevant for many undergraduate students. As one student reflected, "this past year, I just knew so many people who passed away; it was likely because I have grown older and entered college." The undergraduate experience, as this respondent explained, is a transition into adulthood and is accompanied by deeply personal and sometimes tragic experiences. As one student remarked, the course "made me think about deep existential questions" and was shocked by "how much I got out of [the course]." Death becomes more common as the aging process progresses, and college students may experience death in some context. Students also explicitly recognized the benefits of the check-ins and other course pedagogies in terms of their academic liberal arts experience-one student noted "how often [this course] was connected to other conversations and topics I was discussing in my other courses," signifying a potential crossover impact in other classes.

All respondents noted a perceived value in taking a course that discussed death, dying, and palliative care in college. While some respondents felt that the course may be more useful for students "interested in medicine," others remarked on the applicability outside of medicine in forcing undergraduates to "answer deep existential questions about how we should spend our lives." One respondent was "grateful I had the opportunity to take [the course]" to reflect on their own life experience. Another noted the value of the course in allowing "me to explore topics, questions, and aspects of the human experiences that I otherwise would not have been able to. I think it was an enormously impactful experience and would highly recommend the course to anyone interested in stepping outside [of] their comfort zone and exploring something not commonly discussed." As exhibited in the feedback from the course, a willingness to discuss historically taboo and stigmatized topics-like death, dying, and palliative care-is necessary, effective, and productive in the context of a college course.

Ultimately, our three-pronged pedagogical approach to the pilot curriculum, integrated within the Community of Inquiry model, successfully engaged students socially, cognitively, and academically. Faculty and students cooperatively created a conducive environment for challenging and vulnerable conversation. What emerged was not only an improved understanding of palliative care as an interdisciplinary field, but also shifts in individual perspectives, behaviors, and attitudes toward the end of life. The conversations facilitated through a humanistic undergraduate curriculum proved to have an impact on the lived experiences of many healthy emerging adults who were enrolled.

\section{Challenges and limitations}

For practical purposes, there was a concerted choice to center the pilot course on end-of-life care, a piece of the overall complexity that comprises palliative care as a specialty. Taboo and contentious topics tend to garner attention among undergraduate students. As such, we made the choice to gear the course toward end-of-life care, rather than more upstream concepts in caring for patients with serious illness. As such, this risks equating end-of-life care and palliative care among undergraduates enrolled in the course. Though our findings indicate that this was not the case, the focus of the material proved to be a limitation of the pilot course in addressing palliative care generally. A course focused on the holistic applications of palliative care over the course of serious illness care may have alternative outcomes and could perhaps be better suited for future iterations of this course at our institution.

When prompted to reflect on what was most challenging about the course, many students remarked thatwhile the deeply personal element of the course was freeing and "appreciated" - it could be, at the same time, overwhelming and sometimes a barrier to participation in course discussions. Some of the students reflected that the most challenging component of the course was "feeling comfortable enough to share personal aspects of my own life experience and grappling with the themes of end of life and end-of-life care."

Respondents noted a lack of structure and flow to some of the course content and discussions, but also that this lack of structure actually enabled and encouraged exploration inside the classroom through student-led sessions, as exemplified by the diversity of topics covered. Additionally, and as noted by some students and in the literature about grief and anxiety about death, there is likely a limit at which discussing death and dying becomes unbearably upsetting and depressing. Undergraduate students are not morticians or pathologists. Therefore, it is important to define scaffolding within which faculty can be supportive of undergraduate students and understand potential issues of student mental health for those who are encouraged to delve deeply into heavy content surrounding end-of-life issues. Crossing the boundaries into poorly charted territory by developing a curriculum 
in palliative care comes with the challenge of exposing emerging adults to difficult conversation, while also preventing unhealthy mental fatigue.

\section{Limitations of analysis}

Our evaluation and overall assertion that education in palliative care, death, and dying is crucial for development of emerging adults has the following caveats: (1) our course was small and only just over half of the students in the course responded to the survey and (2) not all emerging adults are enrolled in college. Our institution is composed of a diverse but educated population of individuals who attend a 4-year private institution. The success of a course like this may vary at other types of institutions. Additionally, palliative care education may be necessary in communities beyond undergraduate institutions and may even be most relevant and important in communities that cannot easily access formal education.

\section{Conclusions}

Post-secondary institutions foster learning environments in which students openly engage in difficult conversations, learn to challenge one another, and determine their own unique perspectives on political and social issues. Emerging adulthood is a time when individuals must begin making informed decisions about their future healthcare (Berger, 2012, 12). Death is inevitably part of the life-cycle; however, ADs, DPOAHs, and death and dying are not often included in the lesson plan. College environments provide fertile ground for death talk, yet common misperceptions continue to prevent such dialogue. The illusion that depression and anxiety will result from engaging in such conversations proves to be a barrier to completion of an $\mathrm{AD}$, and the larger societal misconception and assumption that "healthy, young adults do not need to consider their own mortality" continues to exist (Sanders \& Robinson, 2017, 332). This mentality is detrimental for the $88 \%$ of healthy emerging adults who want to engage in end-of-life decision-making (Sanders \& Robinson, 2017, 333).

We identified a need and desire for an undergraduate course in death and dying that included facilitating students' reflection on their own death in an academic context. As part of a larger project to establish a collegewide and nationwide engagement of these issues among students and faculty, we created and taught such a course. We developed this educational experience using the three-pronged Community of Inquiry model of social, teaching, and cognitive presence. Within the model we focused on: building a trusting community, creating a course that values and contextualizes personal experiences and beliefs, and, undergirding these two, having instructors model and then facilitate effective scholarship. We deployed particular pedagogical strategies within these themes and showed that, largely, students found the approaches effective and meaningful, that we had indeed established a trusting Community of Inquiry. This is eloquently summed up in one student's reflection on their experience in our course:

Th[is] class has been one of the most thought-provoking classes I have ever taken, for, unlike some other classes, it is deeply personally relevant as a human. It has not merely changed what I think or how I think but how I hope to live. Prior to this class, I hadn't looked at death as an essential and necessary part of life, and especially not death in the United States. I had simply hidden from it. While I won't claim that I am close to understanding death, I think I have a greater appreciation for the way it affects our lives, and am perhaps more receptive to further understandings. This class has also made me consider and reconsider the assumptions which I make about the world, and thus has tried to instill a greater degree of epistemic humility, a trait which appears to be helpful in many, if not most environments. I now am not quite so sure of what I know, or at least am more open to questioning how I know what I know. In all, I think this class has made me more open to cognitive dissonance and less afraid of uncertainty.

We envision other institutions and departments able to adapt pedagogies within our model and these themes appropriate for their needs and institutional context.

\section{Appendices \\ Appendix A: feasibility study, Spring 2019 Methodology and study design}

IRB approval was granted under the IRB protocol submitted in Fall 2019 (IRB00116952). Those who agreed to participate indicated this by signing a consent form. They were then asked a series of questions in three categories: (1) Demographic Information; (2) Familiarity with End-of-Life Communication Tools; and (3) Comfort Discussing the End of Life. Demographic information included age, status as undergraduate student, institution of study, and major. Questions surveyed familiarity with $\mathrm{AD}, \mathrm{DPOAH} /$ health care proxy, and do-not-resuscitate (DNR) orders. Respondents were asked about conversations with healthcare providers, family members, and academic touchpoints, and prior dialogue regarding the 
aforementioned tools. Participants were asked about current comfort discussing end of life, among other questions about palliative care education.

\section{Feasibility study research instrument Section 1: Demographics}

Age? (Between 17- and 26-years-old)

Are you an undergraduate student? Yes/No

If no, are you a graduate student? Yes/No

Do you attend Emory University? Yes/No

If not, where do you attend school?

What year are you? Freshman, Sophomore, Junior,

Senior, Graduate

What is your major/focus of study?

\section{Section 2: End-of-Life Communication Tools}

Advance Directives and Familiarity

Do you know what an advance care directive is? Yes/ No

If yes, briefly describe what it is and how it's utilized. (optional)

Has your primary care physician ever spoken to you about an advance directive? Yes/No

Have your parents/legal guardians ever spoken to you about an advance directive? Yes/No

Have you learned about advance directives in an academic course? Yes/No

Have you completed an advance directive in the past? Yes/No

Durable Power of Attorney (Health Care Proxy) and Familiarity

Do you know what a durable power of attorney (health care proxy) is? Yes/No

If yes, briefly describe what it is and how it's utilized. (optional)

Has your primary care physician ever spoken to you about a durable power of attorney? Yes/No

Have your parents/legal guardians ever spoken to you about a durable power of attorney? Yes/No

Have you learned about durable power of attorney in an academic course? Yes/No

Have you designated a durable power of attorney? Yes/No

Do you know what a Do-Not-Resuscitate (DNR) order is? Yes/No

\section{Section 3: Comfort in Discussing End of Life}

On a scale from 1 to 10 , how difficult is it for you to raise and engage in conversation with your FAMILY about death and dying? (Likert scale, 1-extremely easy/10-extremely difficult)

On a scale from 1 to 10 , how difficult is it for you to raise and engage in conversation with your FRIENDS about death and dying? (Likert scale, 1 -extremely easy/10-extremely difficult)

Have you spoken to your mother about her wishes for the end of life? Yes/No

Have you spoken to your father about his wishes for the end of life? Yes/No

Have your parents/legal guardians spoken to you about YOUR wishes for the end of life? Yes/No If you can remember, at what age did you first realize you could die? (Between 5- and 16-years-old, "I don't remember")

At the current moment, do you feel comfortable talking about death and dying? Yes/No

Do you wish your primary care physician spoke more openly about death and dying? Yes/No

Should death and dying be a component of high school education? Yes/No/Maybe

Briefly explain why it should or should not be a component of high school education. (optional)

Should death and dying be a component of postsecondary education? Yes/No/Maybe

Briefly explain why it should or should not be a component of post-secondary education. (optional)
Appendix B: IDS 205 W pilot course evaluation, Fall 2019 Methodology and study design

All enrolled undergraduate students -18 years of age or older-and previously enrolled in the pilot course were eligible to participate. At the end of the course in Fall 2019, students were emailed an online Google Form-with integrated IRB consent documentationand asked to participate at their convenience alongside course evaluations. Students were recruited both during class and sent a targeted recruitment email. Completion of the online form was voluntary and anonymous, and respondents were instructed that completing the evaluation would have no impact on their final grade. There was no compensation for participating in the study. No identifiers were collected during the online survey questionnaire.

The questionnaire was designed with three core sections: (1) Demographics; (2) Course Feedback; and (3) End-of-Life Communication. The first section inquired 
about academic major, year in school, age, and course enrollment. The second section contained both quantitative and qualitative questions to gauge the efficacy of the course and comfort with material taught. The third section aimed to quantitatively understand terminology used when discussing palliative care and end-of-life issues, as well as personal practices.

\section{Institutional review board process and approval}

An IRB protocol (IRB00116952), including consent documentation, was approved and both authors completed CITI certifications in Sociobehavioral IRB Training and, additionally, Kulp completed Good Clinical Practice Training, prior to collecting data.

\section{Demographics}

Of the 13 students enrolled in the course, seven $(n=7)$ completed the approximately 20-min survey. Respondents ranged in age from 19- to 22-years-old, all enrolled as undergraduate students at Emory and in IDS $205 \mathrm{~W}$ during the Fall 2019 semester. Of the respondents, 1/7 were second-year students, 2/7 were third-year students, and 4/7 were fourth-year students. Majors included American Studies, International Studies, Interdisciplinary Studies, Business Administration, Chemistry, English \& Creative Writing, Music, and Linguistics.

\section{IDS 205 W evaluation research instrument Section 1: Demographics}

How old are you? (Between 18- and 23-years-old) Are you an undergraduate student at X? Yes/No Are you enrolled in IDS205W for the Fall 2019 semester? Yes/No

What year are you? First, Second, Third, Fourth What is your major?

\section{Section 2: Course Feedback}

Why did you decide to take this course?

What intrigued you, if anything, about the topic after receiving the syllabus?

Had you heard of palliative care before the course? Yes/No

If yes, how was it defined/explained previously? As you've learned it, what is your definition/understanding of palliative care and its tenants now?
Has this course influenced the way in which you interact with your peers? Family? Parents? How so? At the current moment, do you feel comfortable talking about death and dying? Yes/No

Are you more aware of your own mortality after taking this course? How so?

Are you more accepting of your own mortality after taking this course? How so?

What surprised you most about this course?

What was most challenging about this course?

What would you change about this course?

What does it mean to live knowing that you're going to die, and having spoken intimately about it in class?

Do you think a course on palliative care is beneficial for undergraduates? Why or why not?

\section{Section 3: End-of-Life Communication}

Have you been diagnosed with a chronic or lifelimiting condition/illness? Yes/No

Have your parents/legal guardians spoken with you about your wishes for the end-of-life? Yes/No

\section{Advance Care Directives}

Do you know what an advance care directive is? Yes/No

Did you know what an advance care directive was before this course? Yes/No

Has your primary care physician ever spoken to you about an advance directive? Yes/No

Have your parents/legal guardians ever spoken to you about an advance directive? Yes/No

Have you completed an advance directive in the past? Yes/No

\section{Health Care Proxy / Durable Power of Attorney}

Do you know what a durable power of attorney (health care proxy) is? Yes/No

Did you know what a durable power of attorney (health care proxy) was before this course? Yes/No Has your primary care physician ever spoken to you about a durable power of attorney? Yes/No Have your parents/legal guardians ever spoken to you about a durable power of attorney? Yes/No 
Have you designated a durable power of attorney? Yes/No

\section{Do-Not-Resuscitate Order}

Do you know what a Do-Not-Resuscitate (DNR) order is? Yes/No

Did you know what a DNR order was before this course? Yes/No

\section{Conclusion}

Your response has been collected. Thank you for participating in this survey.

\section{Acknowledgements}

This work is supported by a gift from the Arthur Vining Davis Foundations in partnership with the Woodruff Health Sciences Emory Palliative Care Center and the Emory Institute for the Liberal Arts.

\section{Authors' contributions}

$A E, K L$, and DK together organized and taught the course described; DK also gathered and analyzed the bulk of the data and wrote a large part of the manuscript; AE also provided guidance and analysis and wrote portions of and edited the manuscript; LO facilitated the project through mentoring and advice, taught part of the course, and edited and wrote portions of the manuscript: ST and KL edited and wrote parts of the manuscript; TQ is the primary investigator on the greater project of which this work is part, taught part of the course, and edited and wrote portions of the manuscript. All authors read and approved the final manuscript

\section{Funding}

This work was supported by a gift from the Arthur Vining Davis Foundations. The Foundations played no role in the design of the study, nor in the collection, analysis or interpretation of data, nor in the writing of the manuscript.

\section{Availability of data and material}

Raw data were generated at Emory University. Derived data supporting the findings of this study are available from the corresponding author [AE] upon request.

\section{Declarations}

\section{Competing interests}

The authors have no competing or conflicts of interests to declare.

\section{Author details}

${ }^{1}$ Institute for the Liberal Arts, Emory University, Atlanta, GA 30322, USA. ${ }^{2}$ Woodruff Health Sciences Palliative Care Center, Emory University, Atlanta, GA 30322, USA. ${ }^{3}$ Program in Linguistics, Emory University, Atlanta, GA 30322, USA. ${ }^{4}$ Department of Biology and Institute for the Liberal Arts, Emory University, Atlanta, GA 30322, USA.

Received: 3 August 2020 Accepted: 25 October 2021

Published online: 07 November 2021

\section{References}

Arnold, B., Curtis, R., Fischer, J., Fratkin, M., Isaac, M., Lakin, J., Mark, N., Merel, S., McGuire, T., Tulsky, J., Sakata, V., The John A. Hartford Foundation, \& Cambia Health Foundation. (2020). COVID-Ready Communication Skills: A Playbook of VitalTalk Tips. VitalTalk.
Berger, R. S. (2012). Including adolescents and young adults in decisions at the end-of-life. Journal of Student Nursing Research, 5(1), 12-18.

Billings, J. A. (1997). Palliative care in undergraduate medical education. Status report and future directions. JAMA: the Journal of the American Medical Association, 278(9), 733-738. https://doi.org/10.1001/jama. 278.9.733

Cha, A. E., \& Janes, C. (2020). Young adults are also affected by Kawasaki-like disease linked to coronavirus, doctors say. The Washington Post. https:// www.washingtonpost.com/health/2020/05/21/misc-c-kawasaki-coron avirus-young-adults/

Chriscaden, K. (2017). More than 1.2 million adolescents die every year, nearly all preventable. World Health Organization. https://www.who.int/newsroom/detail/16-05-2017-more-than-1-2-million-adolescents-die-everyyear-nearly-all-preventable

Dehlin, L., \& Reg, L. M. (2009). Adolescents' experiences of a parent's serious illness and death. Palliative and Supportive Care, 7(1), 13-25. https://doi. org/10.1017/S1478951509000042

Duke, N. N., Borowsky, I. W., Pettingell, S. L., Skay, C. L., \& McMorris, B. J. (2011). Adolescent early death perception: Links to behavioral and life outcomes in young adulthood. Journal of Pediatric Health Care, 25(4), 224-234. https://doi.org/10.1016/j.pedhc.2010.03.004

Garrison, D. R., \& Akyol, Z. (2013). The Community of Inquiry theoretical framework. In M. G. Moore (Ed.), Handbook of distance education (3rd ed., pp. 104-119). New York: Routledge.

Head, B., Chenault, J., Earnshaw, L., Pfeifer, M., Sawning, S., Schapmire, T., \& Shaw, M. (2016). Improving medical graduates' training in palliative care: Advancing education and practice. Advances in Medical Education and Practice. https://doi.org/10.2147/AMEP.S94550

Howard, J. (2018). A "wake-up call" about what's killing America's young people. CNN.Com. https://www.cnn.com/2018/06/01/health/youth-injurydeath-rate-cdc-study/index.html

Jalmsell, L., Kontio, T., Stein, M., Henter, J.-I., \& Kreicbergs, U. (2015). On the child's own initiative: Parents communicate with their dying child about death. Death Studies, 39(2), 111-117. https://doi.org/10.1080/07481187. 2014.913086

Kavalieratos, D., Ernecoff, N., \& Keim Malpass, J. (2014). "I think I am a real person": How do healthy young adults view advance care planning? (S734). Journal of Pain and Symptom Management, 47(2), 485-486. https://doi. org/10.1016/j.jpainsymman.2013.12.152

Kavalieratos, D., Ernecoff, N. C., Keim-Malpass, J., \& Degenholtz, H. B. (2015). Knowledge, attitudes, and preferences of healthy young adults regarding advance care planning: A focus group study of university students in Pittsburgh, USA. BMC Public Health, 15(1), 197. https://doi.org/10.1186/ s12889-015-1575-y

Kon, A. A. (2006). Assent in pediatric research. Pediatrics, 117(5), 1806-1810. https://doi.org/10.1542/peds.2005-2926

Lyckholm, L., \& Kreutzer, K. O. (2010). Creating training opportunities in pediatric palliative care. American Medical Association Journal of Ethics, 12(7), 548-553. https://doi.org/10.1001/virtualmentor.2010.12.7.medu2-1007

Noppe, I. C., \& Noppe, L. D. (2004). Adolescent experiences with death: Letting go of immortality. Journal of Mental Health Counseling, 26(2), 146-167. https://doi.org/10.17744/mehc.26.2.py2tk0kmay1ukc3v

Odeniyi, F., Nathanson, P. G., Schall, T. E., \& Walter, J. K. (2017). Communication challenges of oncologists and intensivists caring for pediatric oncology patients: A qualitative study. Journal of Pain and Symptom Management, 54(6), 909-915. https://doi.org/10.1016/j.jpainsymman.2017.06.013

Pao, M., \& Mahoney, M. R. (2018). Will you remember me? Child and Adolescent Psychiatric Clinics of North America, 27(4), 511-526. https://doi.org/10. 1016/j.chc.2018.05.001

Roadside Theater. (2014). Roadside Theater. About: Story Circles. https://roads ide.org/asset/about-story-circles

Robinson, E. L., Hart, B., \& Sanders, S. (2019). It's okay to talk about death: Exploring the end-of-life wishes of healthy young adults. Death Studies, 43(6), 389-396. https://doi.org/10.1080/07481187.2018.1478913

Sanders, S., \& Robinson, E. L. (2017). Engaging college undergraduates in advance care planning. OMEGA - Journal of Death and Dying, 74(3), 329-344. https://doi.org/10.1177/0030222815598912

Slaughter, V., \& Griffiths, M. (2007). Death understanding and fear of death in young children. Clinical Child Psychology and Psychiatry, 12(4), 525-535. https://doi.org/10.1177/1359104507080980 
Tripken, J. L., \& Elrod, C. S. (2018). Young adults' perspectives on advance care planning. American Journal of Hospice and Palliative Medicine, 35(4), 627-634. https://doi.org/10.1177/1049909117727456

Tulsky, J. A. (1998). Opening the black box: How do physicians communicate about advance directives? Annals of Internal Medicine, 129(6), 441. https:// doi.org/10.7326/0003-4819-129-6-199809150-00003

What should the age range be for AYA oncology? (2011). Journal of Adolescent and Young Adult Oncology, 1(1), 3-10. https://doi.org/10.1089/jayao.2011. 1505

Wiener, L., Zadeh, S., Battles, H., Baird, K., Ballard, E., Osherow, J., \& Pao, M. (2012). Allowing adolescents and young adults to plan their end-of-life care. Pediatrics, 130(5), 897-905. https://doi.org/10.1542/peds.2012-0663

Wiener, L., Zadeh, S., Wexler, L. H., \& Pao, M. (2013). When silence is not golden: Engaging adolescents and young adults in discussions around end-of-life care choices. Pediatric Blood \& Cancer, 60(5), 715-718. https://doi.org/10. $1002 / p b c .24490$

Willis, C. A. (2002). The grieving process in children: Strategies for understanding, educating, and reconciling children's perceptions of death. Early Childhood Education Journal, 29(4), 221-226.

World Health Organization. (2018). Adolescents: Health risks and solutions [Health]. World Health Organization. https://www.who.int/en/newsroom/fact-sheets/detail/adolescents-health-risks-and-solutions

\section{Publisher's Note}

Springer Nature remains neutral with regard to jurisdictional claims in published maps and institutional affiliations.
Ready to submit your research? Choose BMC and benefit from:

- fast, convenient online submission

- thorough peer review by experienced researchers in your field

- rapid publication on acceptance

- support for research data, including large and complex data types

- gold Open Access which fosters wider collaboration and increased citations

- maximum visibility for your research: over $100 \mathrm{M}$ website views per year

At BMC, research is always in progress.

Learn more biomedcentral.com/submissions 